\title{
Thyroid Surgery during COVID-19 Pandemic - Principles and Philosophies
}

\author{
Ashok R. Shaha, $\mathrm{MD}^{1}$ \\ ${ }^{1}$ Head \& Neck Service. Memorial Sloan Kettering Cancer Center. New York
}

April 28, 2020

\section{INTRODUCTION}

We are quite familiar with the COVID-19 epidemic and its unprecedented implications. It has clearly changed our lives, healthcare, clinical practice, urgency of the health problems, financial implications and mental health. The issues of mental health are applicable both to the patients and healthcare providers. Obviously, we need to pay special attention to the patients suffering from COVID-19 especially those who are symptomatic or having major health crisis such as pulmonary issues and multiorgan failure.

As of the $8^{\text {th }}$ of April, globally there have been approximately 1.52 million confirmed cases of COVID-19 of whom 90,000 are dead. In the United States, the confirmed COVID-19 cases are reported to be 435,564 while the reported deaths are 14,829. New York State has faced the major brunt of this pandemic with confirmed cases of 147,037 and death number of 6,220 .

In spite of this major health crisis patients are always concerned about their own problems in relation to other health issues especially with fear of proven or suspicious cancers. Clearly, some of the cancers are life-threatening and will require urgent attention while other tumors may be monitored or treated at a later date when the COVID-19 issues are relatively settled. In a referral center or a tertiary care cancer center it is fairly common to receive consultations regarding thyroid problems or thyroid tumors.

Even though, there are no set guidelines in the management of patients asking for thyroid surgery it would be appropriate to manage these patients based on the risk group analysis and the overall risks of progression to life-threatening issues. We need to explain every patient that thyroid tumors grow slowly and there is no need for active and emergent intervention. It is quite appropriate to wait for 4-6 months.

If the patient is extremely anxious a follow up ultrasound may be performed in 3-4 months to document the stability of thyroid tumor. We have divided thyroid cancer patients for almost 50 years into low, intermediate and high-risk groups based on their prognostic features ${ }^{1}$. We popularly described this as good, bad and ugly tumors. The prognostic factors were described as age, grade of the tumor, size of the tumor, extrathyroidal extension, distant metastases, etc. Other prognostic factors such as multiple lymph node metastases and the molecular analysis should go into the equation of management of these patients. Needless to say, patients are extremely concerned for the fear of any cancer whether it is thyroid or pancreatic cancer. It is our responsibility to explain to the patients the concern about these cancers on their overall prognosis and the best timeline definition for active intervention. The new American Thyroid Association guidelines published in 2015 have done a fantastic job in line with the biology of these tumors and appropriate management ${ }^{2}$. As a matter of fact, the ATA endorsed observation as a definitive approach in proven microcarcinomas. This clearly reflects the management of these tumors in relation to their biology and avoiding over treatment. Let the punishment fit the crime or let the treatment not be worse than the disease is quite appropriately applied to thyroid cancer. However, it would be important to define certain indications and road map of active management of some these thyroid cancers. If we use the analogy of management of thyroid cancer during 
pregnancy and delaying the treatment by 9-10 months, it would be the same philosophy of managing these patients during the COVID-19 pandemic. Clearly, some patients will require urgent or active intervention in a timely fashion. The following summary will describe some of the decision-making issues.

- Anaplastic Thyroid Cancer - patients with rapidly growing thyroid tumors with proven anaplastic thyroid cancer will obviously require emergent management. The decision regarding surgical intervention should be made based on the extent of the disease and cross-sectional imaging. Appropriate BRAF based therapies and external radiation therapy should be implemented. If the tumor appears to be unresectable there is no reason to bring these patients to the operating room. The definitive diagnosis could easily made with ultrasound guided core biopsy, and appropriate immunohistochemistry. The issue of airway management is always a difficult problem in anaplastic thyroid cancer and more so during COVID-19 pandemic. Obviously, testing the patient for Covid-19 is important since patient may require either active airway intervention or hospitalization with concern of exposing healthcare workers. As mentioned in the first anaplastic thyroid cancer guidelines, elective tracheostomy is best avoided however may be necessary if the patient is having acute airway distress ${ }^{3}$. A due consideration should be given to controlled cricothyrotomy.

- Medullary Thyroid Cancer - Appropriate evaluation of extent of the disease with calcitonin, CEA, ultrasound and cross-sectional imaging is very important before consideration of timely surgical intervention. If the disease appears to be limited and calcitonin levels are not high (under 400) patients can be monitored for a few months without surgical intervention hoping for COVID-19 peak to settle. Generally, medullary carcinoma is a chronic disease and observation with close monitoring would be quite appropriate until the social circumstances get better. Obviously, this will require extensive discussion with the patient and the family which can be easily done even by phone conversations or Facebook. A discussion directly by responsible attending surgeon would give a lot of confidence to the patient and the family. They need to understand that waiting for the best time for surgery is unlikely to hurt them or lead to major progress of the disease. The prognosis essentially would remain the same.

- Locally Aggressive Thyroid Cancer - These are the patients who will require detailed evaluation of the extent of the disease, its involvement in relation to the central compartment vital organs such as recurrent laryngeal nerve, trachea, esophagus, and major vascular structures. Appropriate crosssectional imaging will be of great help. If patient does require fiberoptic evaluation it would be best done with the hospital guidelines and appropriate protection to the healthcare staff. Obviously, COVID-19 testing would be important prior to any active intervention. The decisions about surgery in light of COVID-19 pandemic would be quite critical as to how long we can delay the surgical procedure without compromising the total surgical resection and encroachment on vital central compartment structures. The decisions may be slightly different if the preoperative FNA has resulted in poorly differentiated thyroid cancer. It would be quite appropriate to discuss some of these cases with our colleagues in multidisciplinary team since we are able to hold virtual tumor boards. Avoiding surgical compromise is important in these patients however waiting for a reasonable time would not be inappropriate.

- Patients with Large Primary Tumors and Bulky Nodal Disease - The history of the presence of tumor and the duration of the nodal metastasis would be quite helpful to project the best timing of surgery in these patients. Again, appropriate cross-sectional imaging and approximation of the tumor to the vital structures is critical in making the best decision regarding appropriate timing of surgery in these patients.

- Low and Intermediate Risk Thyroid Carcinomas - These patients can wait for surgery for a period of time (3-6 months) until we have a better handle on COVID-19, and they are not a risk to the healthcare workers. If the patients need extended period of observation, a repeat imaging with ultrasound in 3-4 months will encourage the patients to delay the surgery further.

- Microcarcinomas - As reported by a large series of patients from Kobe, Japan; Sloan Kettering, these patients with microcarcinomas can definitely be observed ${ }^{4,5}$. Most of these patients can be encouraged not only to delay the surgery but to remain under active surveillance or deferred intervention. Again, appropriate ultrasound will define the exact location of the disease and need of active intervention. 
- Recurrent Thyroid Carcinoma - The majority of the recurrences especially in the central compartment nodes or lateral neck nodes are essentially the persistent diseases. They could be observed for an extended period of time with repeat imaging studies in 4-6 months. The only time one would consider active surgical intervention, if the tumor is plastered against the trachea for the fear of future encroachment into the trachea. Alternate treatment choices such as alcohol injection, radio frequency ablation may be considered for localized nodal recurrences.

- Indeterminate Thyroid Nodules - most of these patients will be in the group of Bethesda III and IV categories. These patients can be easily monitored and if the tumors are small even if they're BRAF or TERT positive, could be monitored for a period of time before active surgical intervention. The positivity of the molecular markers and the quantification of the risk of malignancy is not a determinate for emergent surgical intervention.

- Large Goiters - the majority of the large goiters have generally been there for a long period of time and surgery could be easily avoided even with tracheal deviation and mild compression unless there is a rapid progression, major compression symptoms or impending acute airway issues.

- Benign Thyroid Conditions - benign thyroid nodules, Hashimoto's thyroiditis, or Graves' disease could be managed appropriately as before and probably may not be in-person consultation. The majority of these patients can be easily consulted on telephone, Skype or Facetime which will give patients a sense of confidence and make them feel that the treating physician is actively involved in their care and follow up. The guidelines recommended by ATA for fine needle aspirations of incidental thyroid nodules should be applied vigorously. It would be best to avoid FNA on smaller and nonsuspicious thyroid nodules.

- Moral Dilemma - I am sure there will be many discussion points in above recommendations. These are not written in any of the textbooks or guidelines. These are clinical observations during the early period of COVID-19 pandemic. Hopefully, God willing, the pandemic will be over soon, and we will go back to our regular clinical practices. However, until then, it is our responsibility to manage our patients best, give them a full sense of confidence and avoiding major progression of their tumors and life-threatening issues. We also have a responsibility to the healthcare workers who take the major brunt of exposing themselves to the COVID-19 which may become lethal in a few individuals. This definitely raises a major new dilemma to the healthcare workers. Every profession has certain risks and concerns. For example, a frontline army personnel, a firefighter, or a policeman where both the individuals and their families are aware about the life-threatening risks. However, until the COVID-19 pandemic occurred nobody realized the life-threatening risks to the healthcare workers. This clearly creates a major social and ethical dilemma amongst healthcare workers and their families. Even though the non-essential staff can work from home, the essential staff such as frontline healthcare workers have to be exposed themselves to proven and unproven COVID-19 patients. This may lead to major ethical issues and mental depression amongst healthcare workers. What would be the answer to the 10-yearold child when he tells his father, "Dad, please don't go to work. I'm afraid you may catch COVID-19 and you are the only one I have."

We don't have the answers to these questions, however, I would like to salute the frontline healthcare workers who have been actively involved in offering the best medical care to the patients suffering from COVID-19 and offering them and the society a Glimpse of Hope. These are the true Noble Laureates.

\section{References:}

1. Shaha, AR. Implications of prognostic factors and risk groups in the management of differentiated thyroid cancer. Laryngoscope. 2004, 114; 393-402.

2. Haugen BR, Alexander EK, Bible KC, Doherty GM, Mandel SJ, Nikiforov YE, et al. 2015 American Thyroid Association - Management guidelines for adult patients with thyroid nodules and differentiated thyroid cancer; the American Thyroid Association Guidelines Task Force on Thyroid nodules and differentiated thyroid cancer. Thyroid. 2016, 26; 1-133.

3. Smallridge RC, Ain KB, Asa SL, Bible KC, Brierley JD, Berman KD et al, American Thyroid Association Guidelines for Management of patients with anaplastic thyroid cancer. Thyroid. 2012, 22; 
1104-39.

4. Miyauchi, A. Clinical trials of active surveillance of papillary microcarcinoma of the thyroid. World J Surg. 2016, 40; 516-22.

5. Tuttle, RM, Fagin JA, Minkowitz G, Wong RJ, Roman B, Patel S et al, Natural history and tumor volume kinetics of papillary thyroid cancers during active surveillance. JAMA Otolaryngol Head Neck Surg. 2017, 143; 1015-1020. 